\title{
Einzelbewerber bei den Bundestagswahlen von 1949 bis 2013: zahlreich, aber chancenlos
}

\author{
Christian Nestler
}

\section{Deutschland: ein Parteienstaat}

Deutschland ist ein Parteienstaat. ${ }^{1}$ Diese Feststellung ist in keiner Weise pejorativ, sondern charakterisiert prägnant die Stellung der Parteien im politischen System der Bundesrepublik. Ihre Prominenz ist hinreichend mit Artikel 21 des Grundgesetzes (GG) belegt. Die Realität ihrer Bedeutung spiegelt sich im Umfang der wissenschaftlichen Beschäftigung mit dem Thema. ${ }^{2}$ Einschränkend ist zu sagen, dass sich dieses Interesse fast ausschließlich auf die etablierten, also den in Parlamenten vertretenen Parteien richtet.

In der Meinungsforschung und der medialen Wahlberichterstattung werden alle nicht etablierten Parteien unter dem Sammelbegriff, „Sonstige“ subsumiert. Ausnahme waren seit 2011 auf der Bundesebene die Piratenpartei (Piraten) und die Alternative für Deutschland (AfD). Im regionalen Kontext gibt es, von den Genannten abgesehen, beispielsweise mit der Deutschen Volksunion (DVU), der Nationaldemokratischen Partei Deutschlands (NPD) und den Freien Wählern (FW) weitere Abweichungen von dieser Regel. Im Unterschied zum hier behandelten Untersuchungsgegenstand werden jene allerdings nach einem anfänglichen Erfolg in die Umfragen und Darstellungen mit einbezogen. ${ }^{3}$

Parteilose Einzelpersonen - in der amtlichen Bezeichnung: Einzelbewerber - finden sich in den präsentierten Grafiken der Meinungsforschung nicht wieder. Das resultiert schon

1 Es sei nur auf eine kleine Auswahl von Schriften, die sich auch auf das Konzept vom und die Kritik am Parteienstaat beziehen, verwiesen: Klaus von Beyme, Die politische Klasse im Parteienstaat, Frankfurt am Main 1993; Wilhelm Hennis, Auf dem Weg in den Parteienstaat. Aufsätze aus vier Jahrzehnten, Stuttgart 1998; Anna Bettina Kaiser (Hrsg.), Der Parteienstaat. Zum Staatsverständnis von Gerhard Leibholz, Baden-Baden 2013; Gerhard Leibholz, Strukturprobleme der modernen Demokratie, Karlsruhe 1958; Franz Walter, Vom Milieu zum Parteienstaat. Lebenswelten, Leitfiguren und Politik im historischen Wandel, Wiesbaden 2010. Dabei ist festzuhalten, dass zumeist der Parteienstaat als negative Entwicklungsperspektive der Parteiendemokratie gezeichnet wird. Hierzu beispielhaft Gotthard Breit / Peter Massing, Krise des Parteienstaates?, in: Politische Bildung, 43. Jg. (2009), H. 1, S. 162 - 169; Oscar W. Gabriel / Oskar Niedermayer / Richard Stöss (Hrsg.), Parteiendemokratie in Deutschland, Wiesbaden 2002; Antonius Liedhegener / Torsten Oppelland (Hrsg.), Parteiendemokratie in der Bewährung. Festschrift für Karl Schmitt, BadenBaden 2009. In diesem Artikel wird die Entwicklung der Einzelbewerber daher, in Anlehnung an Wilhelm Hennis, Der ,Parteienstaat' des Grundgesetzes. Eine gelungene Erfindung, in: ders., a.a.O. (Fn. 1), S. 107 - 135, als Aspekt der postulierten Krise des Parteienstaats verstanden.

2 Die Darstellung der gesamten Literatur mit Bezug zum Thema „Parteien in Deutschland“ rechtfertigt eine eigene - wahrscheinlich mehrbändige - Publikation. Selbst eine oberflächliche Recherche im Katalog der Deutschen Nationalbibliothek ergibt ohne Zeitschriftenaufsätze mehr als 1.500 Treffer.

3 Das Beispiel der Piratenpartei zeigt aber auch, wie schnell diese Wahrnehmungsebene wieder verlassen wird. So hat unter anderem die Forschungsgruppe Wahlen im April 2013, mit der Gründung der Alternative für Deutschland (AfD), den „Piratenslot“ mit der neuen Partei besetzt. Nach der Bundestagswahl 2013 haben die meisten Meinungsforschungsinstitute diesen Schritt ebenfalls nachvollzogen. Nachvollziehbar auf einen Blick bei: http://www.wahlrecht.de/umfragen/index.htm (Abruf am 29. Oktober 2014). 
aus der Reduzierung der Auflistung auf die Zweitstimmenergebnisse. Als unabhängiger Kandidat wirbt man in seinem Wahlkreis nur um Erststimmen für den Gewinn des Direktmandats.

Einzelbewerber sind, gemäß dem immer noch gültigen ${ }^{4}$, im Zusammenhang mit Kleinstparteien von Richard Stöss 1975 geäußerten Diktum, eine „terra incognita“ der politikwissenschaftlichen Forschung in Deutschland. ${ }^{5}$ Das überrascht insofern, als international beispielsweise parteilose Staatspräsidenten keine Seltenheit sind ${ }^{6}$; mit Joachim Gauck besetzt seit 2013 erstmals ein parteiloser das höchste deutsche Staatsamt. ${ }^{7}$ Darüber hinaus gibt es eine steigende Zahl von Bürgermeistern ${ }^{8}$ nach diesem Muster. Auf der lokalen Ebene ist Parteienzugehörigkeit allein kein Erfolgsgarant ${ }^{9}$; vielmehr spielen hier seit der Wiedervereinigung Wählergemeinschaften eine große Rolle ${ }^{10}$, setzt doch schon die süddeutsche Ratsverfassung - in Deutschland die am häufigsten anzutreffende Kommunalverfassung auf ein tendenziell parteiunabhängiges Modell. ${ }^{11}$ Mit Bezug auf andere westeuropäische Demokratien wurde daher bereits die Frage gestellt, ob ein Zeitalter von nicht parteigebundenen Akteuren angebrochen sei. ${ }^{12}$ Diese Überlegung resultiert aus den für Staatspräsidenten und die kommunale Ebene skizzierten Erfolgen und der Feststellung, dass die Zustim-

4 Vgl. Uwe Kranenpohl / Oskar Niedermayer, Kleinstparteien, in: Oskar Niedermayer (Hrsg.), Handbuch Parteienforschung, Wiesbaden 2013, S. 663 - 681, S. 678. Die Autoren, ebenda, S. 679, sprechen von „allenfalls graduellen Veränderungen“ gegenüber der Feststellung von 1975.

5 Vgl. Richard Stöss, Terra incognita der Parteienforschung. Splitterparteien in der Bundesrepublik, in: ZParl, 6. Jg. (1975), H. 2, S. 254 - 266. Auch die Analysen der Bundestagswahl 2013 nahmen die Einzelbewerber nicht in den Blick: Eckhard Jesse / Roland Sturm (Hrsg.), Bilanz der Bundestagswahl 2013. Voraussetzungen, Ergebnisse, Folgen, Baden-Baden 2014; Oskar Niedermayer (Hrsg.), Die Parteien nach der Bundestagswahl 2013, Wiesbaden 2015; Hans Rattinger I Sigrid Roßteutscher / Rüdiger Schmitt-Beck / Bernhard Weßels / Christof Wolf (Hrsg.), Zwischen Fragmentierung und Konzentration. Die Bundestagswahl 2013, Baden-Baden 2014.

6 Das kurioseste Beispiel in dieser Hinsicht ist vermutlich Wladimir Putin, der zwar Vorsitzender der Partei „Einiges Russland“ ist, aber nicht deren Mitglied. Vgl. Thomas Wiede, Putin wird parteiloser Vorsitzender, in: Handelsblatt online vom 15. April 2008, http://www.handelsblatt.com/ politik/international/russland-putin-wird-parteiloser-parteivorsitzender/2946694.html (Abruf am 18. Januar 2014).

7 Dem Bundespräsidenten wird gemeinhin eine Überparteilichkeit zugeschrieben; das ändert jedoch nichts daran, dass Gauck der erste Präsident ohne Parteibuch in der Bundesrepublik seit 1949 ist. Vgl. Robert Christian van Ooyen / Martin H. W. Möllers (Hrsg.), Der Bundespräsident im politischen System, Wiesbaden 2012.

8 Vgl. Patrick Fink, Parteilos, unabhängig, erfolgreich. In Deutschland gibt es immer mehr parteilose Bürgermeister, in: Politik \& Kommunikation, 10. Jg. (2011), H. 6, S. 14 - 15.

9 Vgl. Martin Morlok / Thomas Poguntke / Jens Walther (Hrsg.), Politik an den Parteien vorbei. Freie Wähler und Kommunale Wählergemeinschaften als Alternative, Baden-Baden 2012; Ingo Naumann, Wählergemeinschaften in einer Parteiendemokratie. Ihre Stellung im politischen System der Bundesrepublik Deutschland, Wiesbaden 2012.

10 Vgl. Stefan Göhlert / Everhard Holtmann / Adrienne Krappidel / Marion Reiser, Independent Local Lists in East and West Germany, in: Marion Reiser / Everhard Holtmann (Hrsg.), Farewell to the Party Model? Independent Local Lists in East and West European Countries, Wiesbaden 2008, S. $127-148$, S. 127.

11 Vgl. Jörg Bogumil / Lars Holtkamp, Kommunalpolitik und Kommunalverwaltung. Eine policyorientierte Einführung, Wiesbaden 2006.

12 Vgl. Rüdiger Schmitt-Beck / David M. Farrell, Introduction: The Age of Non-Party Actors?, in: dies. (Hrsg.), Non-Party Actors in Electoral Politics. The Role of Interest Groups and Independent Citizens in Contemporary Election Campaigns, Baden-Baden 2008, S. 13 - 24. 
mung für Parteien in zahlreichen Demokratien niedrig ist. ${ }^{13}$ Jene Umfragewerte lassen zusammen mit einem mehr oder weniger ausgeprägten Anti-Parteien-Affekt ${ }^{14}$, der nicht zuletzt durch einen „ausufernden“ Parteienstaat „sekundiert“ wird ${ }^{15}$, die Erfolgsaussichten für Einzelbewerber positiv erscheinen. Gerade in Deutschland könnte diese Logik greifen, wo die wissenschaftliche und populäre Parteienforschung immer wieder Szenarien vom Ende der Mitglieder- und Volksparteien zeichnet. ${ }^{16}$

Diese Ausgangsüberlegungen lassen Fragen nach der Bedeutung von parteilosen Kandidaten relevant erscheinen. Wie entwickelt sich der Umfang der Gruppe der Einzelbewerber bei Bundestagswahlen seit 1949? Finden Anstieg und Abfall ihrer Zahl synchron zu Phasen der Entwicklung des Parteiensystems statt und können sie, wenn auch ohne den Gewinn von Parlamentsmandaten, einen Anstieg ihres Erfolges verbuchen? Da dieses Primärziel nicht erreicht wird, ist zu vermuten, dass es andere Motive für die Wahlteilnahme gibt. Letztlich stellt sich die Frage, welche Erfolgshürden bestehen und inwieweit Parteilose ein Protestventil sind.

\section{Rahmenbedingungen und Motivation von Einzelbewerbern}

\subsection{Der rechtliche Rahmen zur Erlangung eines Sitzes im Deutschen Bundestag}

Der Primat der Parteien in der politischen Willensbildung des Volkes manifestiert sich nicht nur in Art. 21 GG. So enthielt keine Fassung des Parteiengesetzes seit 1967 - seinem Namen entsprechend - die Termini: Einzelbewerber, parteilos oder unabhängig in dem hier gesuchten Sinn. Im Bundeswahlgesetz (BWahlG) findet sich ebenfalls kein direkter Bezug. Dennoch ist in der staatsrechtlichen Diskussion nicht von einer Monopolstellung der Parteien die Rede. ${ }^{17}$ Tatsächlich sind indirekt oder zumindest nicht ausschließlich auf Parteien bezogene Formulierungen in Art. 38 GG und in den $\$ \$ 15,18,20,26,30$ und 49 BWahlG anzutreffen.

13 Diesem landläufigen Urteil wird zum Teil vehement widersprochen. Siehe hierzu Oskar Niedermayer, Das Märchen von der Parteiverdrossenheit, in: \#ZukunftVolkspartei vom 5. März 2012, http://www.zukunftvolkspartei.de/deutschland/das-marchen-von-der-parteienverdrossenheit. html (Abruf am 30. Januar 2014).

14 Vgl. Frank Decker, Parteien im politischen System der Bundesrepublik Deutschland, in: Andreas Kost / Werner Rellecke / Reinhold Weber (Hrsg.), Parteien in den deutschen Ländern. Geschichte und Gegenwart, München 2010, S. 71 - 99, S. 73.

15 Everhard Holtmann, Der Parteienstaat in Deutschland. Erklärungen, Entwicklungen, Erscheinungsbilder, Bonn 2012, S. 19. Im Besonderen soll hier zusätzlich auf die These der Ausbildung von Kartellparteien verwiesen werden. Als Reaktion sehen Richard S. Katz / Peter Mair, Changing Models of Party Organization and Party Democracy. The Emergence of the Cartel Party, in: Party Politics, 1. Jg. (1995), H. 1, S. 5 - 28, S. 23 ff. den Aufstieg von populistischen Parteien, die sich dann gegen den „Block“ stellen. Eine Alternative ist hier offensichtlich die Parteilosigkeit.

16 Vgl. Alexander Gallus / Thomas Schubert / Tom Thieme, Deutsche Kontroversen - Eine Einführung, in: dies. (Hrsg.), Deutsche Kontroversen. Festschrift für Eckhard Jesse, Baden-Baden 2013, S. 9 - 41, S. 24 f. Aktuell auch zu alternativen Betätigungsfeldern abseits der Parteien Oskar Niedermayer (Hrsg.), Abkehr von den Parteien. Parteiendemokratie und Bürgerprotest, Wiesbaden 2013.

17 Vgl. Philip Kunig, Art. 21. Politische Parteien, in: ders., (Hrsg.), Grundgesetz. Kommentar, Bd. 1: Präambel bis Art. 69, München 2012, S. 1482 - 1541. 
So kommt Art. 38, Abs. 2 GG ohne die Nennung von Parteien aus. Konkret heißt es dort zu der Frage, wer gewählt werden kann: „[...] wählbar ist, wer das Alter erreicht hat, mit dem die Volljährigkeit eintritt.“ In geringerem Maße ist zusätzlich Art. 48, Abs. 2 GG von Belang: „Niemand darf gehindert werden, das Amt eines Abgeordneten zu übernehmen und auszuüben. Eine Kündigung oder Entlassung aus diesem Grund ist unzulässig." Einzelpersonen sind also nicht per se von der Erlangung parlamentarischer Mandate ausgeschlossen.

Das entsprechende Bundesgesetz konkretisiert diese Feststellung. Im BWahlG wird in $\$ 4$ zunächst grundsätzlich festgestellt, dass jeder Wähler zwei Stimmen hat. Während die zweite ganz eindeutig in Richtung einer Partei zielt (selbst Wählergemeinschaften sind hier ausgeschlossen $)^{18}$, ist die erste Stimme an eine Person und damit nicht zwangsläufig an eine Partei gebunden. $\$ 5$ ergänzt entsprechend, dass in jedem Wahlkreis ein Abgeordneter gewählt wird. Die wesentlichen Aussagen finden sich im dritten Abschnitt „Wahlrecht und Wählbarkeit“. Die in $\$ 15$ festgeschriebene „Wählbarkeit“ paraphrasiert zunächst lediglich Art. 38, Abs. 2 GG. In $\$ 18$, Abs. 1 wird implizit eine Alternative Wahl von Parteien erwähnt: „Wahlvorschläge können von Parteien und nach Maßgabe des $₫ 20$ von Wahlberechtigten eingereicht werden. "Die Einreichung von Kreiswahlvorschlägen muss laut $\$ 19$ spätestens neunundsechzig Tage vor der Wahl bis 18 Uhr erfolgen.

Wie oben zitiert sind die wesentlichen Bestimmungen für Einzelbewerber in $\$ 20$ enthalten. Abs. 1 erklärt zunächst: „Der Kreiswahlvorschlag darf nur den Namen eines Bewerbers enthalten. Jeder Bewerber kann nur in einem Wahlkreis und hier nur in einem Kreiswahlvorschlag benannt werden. Als Bewerber kann nur vorgeschlagen werden, wer seine Zustimmung dazu schriftlich erteilt hat; die Zustimmung ist unwiderruflich." Abs. 2 ergänzt hierzu die Unterstützung, die ein Vorschlag mindestens braucht, um als rechtmäßig anerkannt zu werden: „Andere Kreiswahlvorschläge [als die von Parteien] müssen von mindestens 200 Wahlberechtigten des Wahlkreises persönlich und handschriftlich unterzeichnet sein. Absatz 2 Satz 2 zweiter Halbsatz gilt entsprechend.“ Dieser Verweis bezieht sich darauf, dass die Unterzeichner wahlberechtigt sein müssen und dies bei der Einreichung der Unterstützerunterschriften nachzuweisen ist. Abs. 4 legt weiter fest, dass Wahlvorschläge, die nicht von Parteien kommen, zusätzlich zum Namen ein Kennwort enthalten müssen. Wie noch zu zeigen sein wird, nutzen die meisten unabhängigen Kandidaten dieses, um eine mehr oder weniger politische Botschaft auf den Wahlzetteln zu platzieren. Dies ist möglich, weil das Kennwort entsprechend $\$ 30$, Abs. 2 beziehungsweise 3 auf dem Wahlzettel alphabetisch anzuführen ist. Grundsätzlich ist die Zulassung der Kreiswahlvorschläge in $\$ 26$ geregelt. Eine Ablehnung ist nur gerechtfertigt, wenn der Vorschlag verspätet eingereicht wurde oder wenn er nicht den Anforderungen des BWahlG entspricht.

Die Wahlkampfkostenerstattung wird in $\$ 49 \mathrm{~b}$ geregelt:

(1) Bewerber eines nach Maßgabe der $\$ \$ 18$ und 20 von Wahlberechtigten eingereichten Wahlvorschlages, die mindestens zehn vom Hundert der in einem Wahlkreis abgegebenen gültigen Erststimmen erreicht haben, erhalten je gültige Stimme 2,80 Euro. Die Mittel sind im Bundeshaushaltsplan auszubringen.

(2) Die Festsetzung und die Auszahlung der staatlichen Mittel sind vom Bewerber innerhalb von zwei Monaten nach Zusammentritt des Deutschen Bundestages bei seinem 
Präsidenten schriftlich zu beantragen; danach eingehende Anträge bleiben unberücksichtigt. Der Betrag wird vom Bundestagspräsidenten festgesetzt und ausgezahlt.

(3) Die Vorschriften des Parteiengesetzes über die absolute und relative Obergrenze finden keine Anwendung.

Eine direkte Erwähnung der unabhängigen Kandidaten findet erst durch den Bundeswahlleiter in der Vorbereitung der Bundestagswahl statt. In dessen Bekanntmachung ${ }^{19}$ zur Wahlteilnahme werden Informationen zu unabhängigen Kandidaten bei einer Bundestagswahl unter „II. Wahlteilnahme von Einzelbewerbern“ gegeben. Auf etwa einer A4-Seite wird alles bisher Dargestellte zusammengefasst. Wesentlich ist die folgende Feststellung: „Für Einzelbewerber, also Wahlbewerber, die keine Parteibewerber sind, sondern die von einzelnen Wahlberechtigten oder von Wählergruppen vorgeschlagen werden, enthält das Bundeswahlgesetz hinsichtlich ihrer Aufstellung keine Vorschriften. "20

Gelingt die Zulassung zur Wahl, steht Einzelbewerbern der Wahlkampf bevor. Hier gibt es eine weitere Hürde, die für Parteien zumindest gewohnheitsrechtlich wegfällt: Wahlwerbespots im öffentlich-rechtlichen Fernsehen. Anstelle von bisher nicht vereinheitlichen Regelungen der einzelnen Länder in dieser Frage sei nur der ZDF-Staatsvertrag zitiert $(\$ 11$, Abs. 1): „Parteien ist während ihrer Beteiligung an den Wahlen zum Deutschen Bundestag angemessene Sendezeit einzuräumen, wenn mindestens eine Landesliste für sie zugelassen wurde. "21 Einzelbewerber sind nicht automatisch ausgeschlossen, allerdings gibt es nicht mal eine Kann-Vereinbarung, wie oben für die Parteien zitiert. Falls die finanziellen Mittel vorhanden sind, kann ein parteiloser Kandidat selbstverständlich wie jeder kommerziell Werbende Sendezeit erstehen. Auch das Aufhängen von Wahlplakaten beziehungsweise das Betreiben von Infoständen ist im rechtlichen Rahmen möglich, erfolgt aber ebenfalls auf eigene Kosten.

Wenn der Wahlkampf erfolgreich war, steht im besten Fall an seinem Ende der Mandatsgewinn. ${ }^{22}$ An diesem Punkt kommt die Geschäftsordnung des Bundestages zum Tragen. Einer Einzelperson ist es nicht möglich, eine Fraktion zu bilden ( $\$ 10)$, sondern nur, sich mit anderen Parteilosen oder einer kleinen Partei - etwa die PDS nach der Bundestagswahl 2002 - als Gruppe zusammenschließen. Die Assoziation mit einer bestehenden Fraktion ist auch möglich. Sonst hat ein einzelner Abgeordneter nur begrenzt Chancen, sich ohne die Unterstützung von in der Regel fünf von Hundert Abgeordneten in die parlamen-

19 Vgl. Der Bundeswahlleiter (Hrsg.), Informationen zur Teilnahme an der Bundestagswahl 2013, Stand 19. Juli 2012, S. 7.

20 Ebenda, S. 5.

21 ZDF-Staatsvertrag vom 31. August 1991, in der Fassung des Zwölften Staatsvertrages zur Änderung rundfunkrechtlicher Staatsvertrage (Zwölfter Rundfunkänderungsstaatsvertrag) in Kraft seit 1. Juni 2009. Die Abweichungen in den einzelnen Ländern beziehen sich zumeist auf den Zeitraum, wie lange vor der Wahl die Regelung gilt. Grundsätzlich gilt die für das ZDF zitierte Regelung, via Rundfunkstaatsvertrag $(\mathrm{RStV})$, sowohl für die öffentlich-rechtlichen als auch für die privaten Rundfunk- und Fernsehanstalten.

22 In diesem Fall greift auch Abs. $1 \$ 6$ BWahlG, und die erfolgreiche Wahl eines Einzelbewerbers führt dazu, dass die für eine Partei eingesetzte Zweitstimme dieser Wähler nicht mehr gezählt wird. Vgl. Alexander Schultz, Einzelbewerber-Wahl kann zum Verlust der Zweitstimme führen, in: Mitteldeutsche Zeitung online vom 22. September 2013, http://www.mz-web.de/politik/ wahlrecht-einzelbewerber-wahl-kann-zum-verlust-der-zweitstimme-fuehren, 20642162,243 80666.html (Abruf am 29. Oktober 2014). 
tarischen Prozesse einzubringen. Über Frage- und Rederecht sowie die Mitgliedschaft in Ausschüssen wird ein Mindestmaß an Beteiligungsmöglichkeiten sichergestellt. ${ }^{23}$

\subsection{Die persönliche Motivation - Differenzierung von organisierten und „echten“ Einzelbewerbern}

Dass eine Einzelperson, wenn sie nicht über größere finanzielle Mittel oder einen klingenden Namen verfügt, kaum medial wahrgenommen wird, ist ein Gemeinplatz. Keine lokale Zeitung wird in einer Umfrage einen parteilosen Kandidaten beachten, und Meinungsforschungsinstitute werden ihn, selbst wenn er lokal bekannt ist, keinesfalls in die bundesweite Sonntagsfrage einbeziehen. ${ }^{24}$ Hieraus folgt, dass bei der Stimmenabgabe des Wählers parteilose Kandidaten auf dem Wahlzettel nicht im Sinne einer rationalen Wahlentscheidung zu rechtfertigen sind. Auf eine einfache Formel gebracht: Wenn schon Parteien, die in den Umfragen vorkommen und unter fünf Prozent geführt werden, mit Verlusten rechnen müssen, weil der Wähler sich nicht sicher sein kann, ob seine Stimme im Parlament repräsentiert sein wird, und Parteien, die gar nicht in den Umfragen geführt werden, definitiv Nachteile haben, können unbekannte Einzelbewerber keine Wunder erwarten.

Eine quasi moralische Hürde, die Parteilosigkeit in Deutschland erfolgreich zu praktizieren, zeigt sich daran, dass auf der Landes- und Kommunalebene schon versucht worden ist, bekannte und aussichtsreiche parteilose Bewerber zu vereinnahmen. Das Werben der etablierten Parteien ist dabei für beide Seiten reizvoll, denn politische Inhalte kann der Einzelbewerber nur mit den Parteien umsetzen und diese gewinnen möglicherweise ein mediales Zugpferd. ${ }^{25}$

An dieser Stelle ist es zunächst notwendig, die Differenzierung zwischen organisierten und „echten“ Einzelbewerbern zu erläutern. Entgegen der Wortbedeutung und den bisherigen Ausführungen können Einzelbewerber sich als parteiunabhängiges Netzwerk im Wahlkampf präsentieren. Das Kennwort, das für den Wahlschein anzugeben ist, kann bei verschiedenen Kandidaten absichtlich identisch sein. Hierbei wird durch Absprache, in Einzelfällen wie der „Wählergruppe Frieden“ 1987 bundesweit, eine parteilose Gemeinschaft geschaffen. Überschneidungen entstehen nicht, weil entsprechend nur ein Kandidat mit dem jeweiligen Kennwort pro Wahlkreis antritt. Diese Strategie schafft ein unabhängi-

23 Diese Möglichkeit wurde durch die Klage, des bereits erwähnten Thomas Wüppesahl beim Bundesverfassungsgericht ermöglicht. Vgl. BverfGE 80, 188 - Wüppesahl, http://www.servat.unibe. $\mathrm{ch} / \mathrm{dfr} / \mathrm{bv080188.html} \mathrm{(Abruf} \mathrm{am} \mathrm{29.} \mathrm{Oktober} \mathrm{2014).}$

24 Eine qualitative Recherche zur Berichterstattung von Lokalzeitungen über Einzelbewerber zur Bundestagswahl 2013 hat allerdings ergeben, dass diese zumindest mit einem Artikel, teilweise sogar mit einem Interview bedacht wurden. Der Mehrwert für die Wirkung dieser Kandidaten ist jedoch zum einen nur bedingt nachvollziehbar; zum anderen erlauben es die Kürze und inhaltliche dünne Berichterstattung kaum, einen Einblick in die Motivation der Bewerber zu nehmen.

25 Die Inkorporation eines Parteilosen auf eine Parteiliste wurde unter anderem in Brandenburg vor der Landtagswahl 2005 betrieben. Hierzu Andrea Beyerlein, Bauernpräsident soll für die SPD zur Wahl antreten, in: Berliner Zeitung online vom 3. März 2004, http://www.berliner-zeitung.de/ archiv/mit-folgart-soll-erstmals-parteiloser-auf-landesliste-bauernpraesident-soll-fuer-spd-zurwahl-antreten,10810590,10156596.html (Abruf am 18. Januar 2014). In der Kommunalpolitik gibt es hier das Bild des „auf dem Ticket der Partei fahrenden“ Bewerbers. 
ges Netzwerk von parteilosen Kandidaten, die prinzipiell für die gleichen Ziele eintreten und eine Gruppe oder eine Fraktion bilden könnten, falls einer oder mehrere von ihnen in den Bundestag einzögen. Demgegenüber stehen „echte“26 Einzelbewerber, die mit einer ausschließlich persönlichen Agenda antreten. Diese Unterscheidung wird in Abschnitt 3 mit Abbildung 1 und Tabelle 1 näher thematisiert.

Die periphere Rolle, die parteilose Kandidaten in den Rechtsquellen und der Wahrnehmung durch die Medien spielen, wirft die Frage nach ihren persönlichen Motiven auf. Hier kann zunächst postuliert werden, dass auch im Parteienstaat Deutschland „Unabhängigkeit“ ein Ideal ist, das einen Wert an sich darstellt. ${ }^{27}$ Zusätzlich dürfte Idealismus, der Wille zur Partizipation am politischen System und dessen Wettbewerb auf der Basis von subjektiv als relevant empfundenen Themen eine Rolle spielen. Neben diesen hehren Zielen gibt es weitere, den schwierigen Rahmenbedingungen eher gerecht werdende, Ambitionen. Diese lassen sich als soziale, ökonomische, kommunalpolitische und persönliche Zielsetzungen beschreiben.

All diese Motive stellen in Rechnung, dass es im Bundestagswahlkampf möglich ist, umfassend zu plakatieren. ${ }^{28}$ Soziale Themen sind dann beispielsweise „6.000 € für die Tafeln in Brandenburg“ wie es im Wahlkreis Potsdam - Potsdam-Mittelmark II - TeltowFläming II gefordert wurde. Eine weitestgehend persönliche Motivation findet sich in dem Slogan „Hundefreunde Kreuzberg“ wieder. In Richtung anstehender Kommunalwahlen kann das Engagement als parteiloser Bewerber um ein Bundestagsmandat eine Art Warmlaufen für diesen Wahlgang sein. Mit Blick auf weniger direkte politische Ambitionen lassen sich zahlreiche Beispiele finden:

- „Für feminismusfreie direkte Demokratie und Justizreform“ (Potsdam - Potsdam-Mittelmark II - Teltow-Fläming II);

- „Gegen Arbeitslosigkeit und Rassismus!“ (Prignitz - Ostprignitz Ruppin - Havelland I);

- „Mehr Rechte für Kinder“ (Regensburg).

Diese Auswahl verdeutlicht die Breite des Bewerberspektrums, in dem es auch selbstironische oder satirische Kandidaten gibt, wie etwa „Opa Braveheart“ im Wahlkreis Helmstedt - Norderstedt. Wirtschaftliche Interessen sind ebenfalls anzutreffen: Wenn ein freiberuflicher Handwerker als Einzelbewerber antritt, kann er über seine Wahlwerbung indirekt durch Nennung der eigenen Tätigkeit - potentielle Kunden ansprechen.

Für die anschließende Analyse werden daher folgende Orientierungen von Bewerbern unterschieden: Alternativ/Reform, Anti-Parteien, Direkte Demokratie, Eigenwerbung, Protest und Single-Issue, wobei es zahlreiche Überschneidungen gibt.

26 Im Gegensatz hierzu wären noch „unechte“ Einzelbewerber abzugrenzen. Personen, die kurz vor der Wahl aus Partei X austreten und im Wahlkampf sagen, dass sie nach ihrer Wahl bei Partei Y Mitglied werden. Hier sieht der Wähler - wenn ihm der Vorgang bekannt ist - entweder das eine oder das andere Parteilogo virtuell auf dem Wahlschein neben dem Namen.

27 In einer Studie des European University Institute wird dies für Irland empirisch belegt. Vgl. Nicole Bolleyer / Liam Weeks, The Puzzle of Non-Party Actors in Party Democracy: Independents in Ireland, in: EUI Working Papers SPS, Nr. 2007/12, S. 2.

28 Die Beispiele wurden aus den Kennwörtern der Einzelbewerber zur Bundestagswahl 2013 ausgewählt. Die Aufteilung auf die vier Zielsetzungen vereinfacht die Ausrichtung aufgrund des Versuchs einer Typologisierung. In der Realität gibt es selbstverständlich Überschneidungen. 
Als Resultat kann in Abgrenzung zu etablierten Parteien ${ }^{29}$ und Kleinstparteien ${ }^{30}$ folgende Definition für Einzelbewerber auf der Bundesebene festgehalten werden: Einzelbewerber sind politische Akteure, die aufgrund ihrer rechtlichen, finanziellen, organisatorischen und programmatischen Einschränkungen praktisch nicht im Wettbewerb mit den politischen Parteien um parlamentarische Repräsentation bestehen können. Die Teilhabe an Entscheidungsprozessen und der Auswahl politischen Führungspersonals ist daher nicht gegeben. Nichtsdestotrotz leisten sie durch ihr Engagement einen Beitrag zur politischen Willensbildung in Deutschland. Darüber hinaus nutzen sie den Bundestagswahlkampf als Plattform für die Durchsetzung sozialer, ökonomischer, politischer und persönlicher Ziele auf der lokalen/kommunalen Ebene.

\section{Einzelbewerber bei den Bundestagswahlen seit 1949}

\subsection{Das Abschneiden der Einzelbewerber bei den Bundestagswahlen seit 1949}

Die Entwicklung des deutschen Parteiensystems seit 1945 wird mit der endgültigen Etablierung der Linkspartei in fünf Phasen eingeteilt ${ }^{31}$ : die Formierungs- (1945 bis 1953), Konzentrations- (1953 bis 1976), Transformations- (1976 bis 1994), Stabilisierungs- (1990 bis 2002) und die fluide Phase (seit 2002).

Für Einzelbewerber lässt sich diese Einteilung im Großen und Ganzen übernehmen. Die erste Bundestagswahl war begünstigt durch das Ein-Stimmen-Wahlverfahren, was temporär eine Art Chancengleichheit zwischen Parteilosen und Parteien schuf. Mit 117 Bewerbern wurde die dritthöchste Zahl an parteiunabhängigen Kandidaten bis einschließlich 2013 erreicht. Eduart Edert, Richard Freudenberg und Franz Ott schafften es als unabhängige Kandidaten in den 1. Bundestag. Diese Erfolge beruhten neben den angesprochenen Faktoren auch auf der noch nicht völligen organisatorischen Abdeckung des Wahlgebietes durch die Parteien oder wie im Fall Ederts an wahltaktischen Erwägungen der Parteien. Er war von CDU, FDP und DP als Gegenkandidat zum Bewerber des Südschleswigschen Wählerbundes im Wahlkreis Flensburg aufgestellt worden.

Im Vorfeld der Wahl zum 2. Bundestag wurde ein neues Wahlgesetz verabschiedet, das neben dem Zwei-Stimmen-Wahlverfahren auch den Fall des Ausscheidens unabhängiger Abgeordneter regelte. Da in diesem Fall keine Parteiliste herangezogen werden kann, würde es im entsprechenden Wahlkreis eine Nachwahl geben. ${ }^{32}$ Diese Regelung fand bisher keine Anwendung.

Von 1953 bis 1983 lässt sich von einer Konzentration der Einzelbewerber sprechen. Mit den Ausnahmen 1969 und 1976, als 19 beziehungsweise 17 unabhängige Bewerber antra-

29 Vgl. Ulrich von Alemann, Das Parteiensystem der Bundesrepublik Deutschland, Wiesbaden 2010, S. 11.

30 Vgl. Dirk van den Boom, Politik diesseits der Macht? Zu Einfluß, Funktion und Stellung von Kleinparteien im politischen System der Bundesrepublik Deutschland, Opladen 1999, S. 21; Christian Nestler, Kleinstparteien in Mecklenburg-Vorpommern, in: Martin Koschkar / ders. I Christopher Scheele (Hrsg.), Politik in Mecklenburg-Vorpommern, Wiesbaden 2013, S. 169 185, S. 172.

31 Vgl. Ulrich von Alemann, a.a.O. (Fn. 29), S. $45-86$.

32 Vgl. BGBl., Jahrgang 1953, Teil I, Nr. 32, S. 470 - 478, S. 477. 


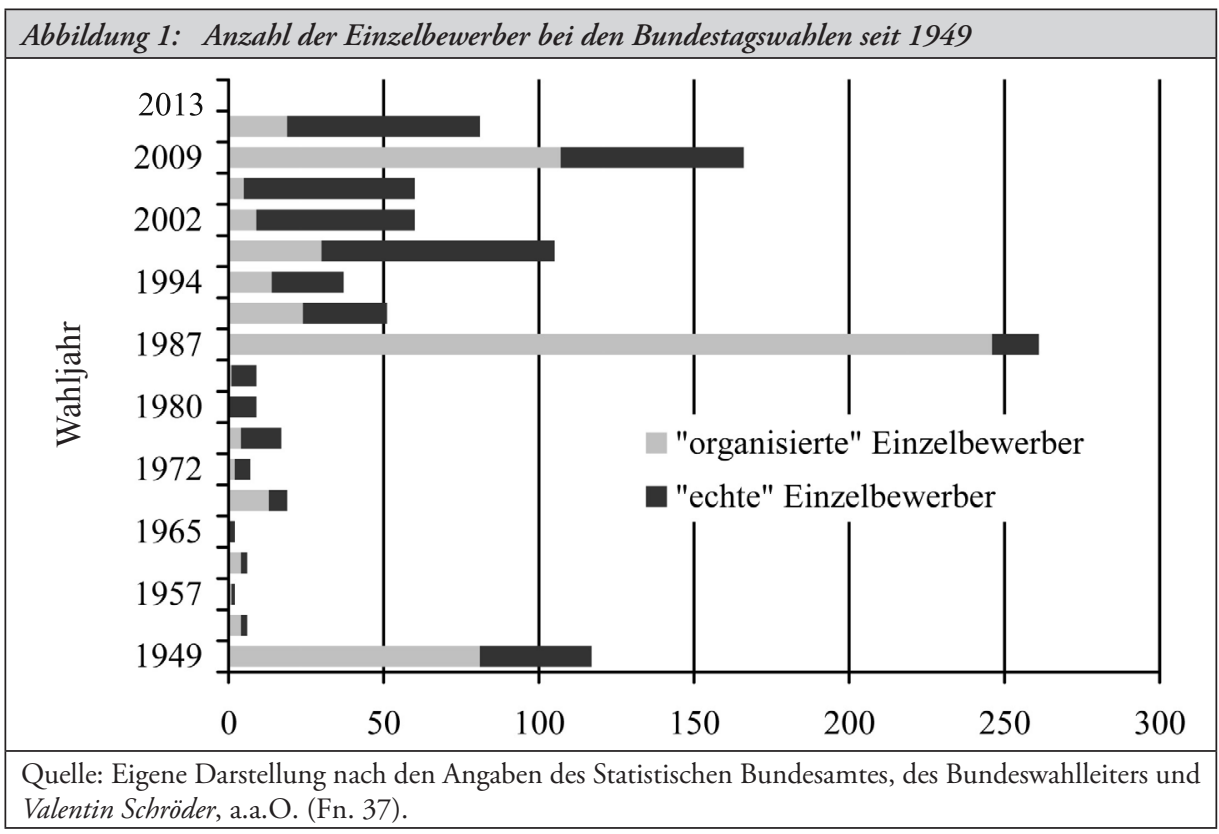

ten, lag ihre Zahl zwischen zwei und neun (siehe Tabelle 1). Lediglich Richard Freudenberg (1953) und Wilhelm Daniels (1969) gelang es mit jeweils über 20 Prozent der Erststimmen, als ernsthafte Bewerber für ein Direktmandat in Erscheinung zu treten.

Ab 1987 wirkte sich die Transformation des deutschen Parteiensystems in Reaktion auf die Etablierung der Grünen auf die Bewerbung von Einzelpersonen aus. Waren 1983 lediglich neun Bewerber angetreten, so waren es vier Jahre später 261. Davon gehörten 245 der „Friedensliste“ an. Diese war ein von der DKP dominierter Versuch, die inzwischen von der ideologisch/programmatischen Ausdifferenzierung der Grünen enttäuschten Wählern abzuwerben. ${ }^{33}$ Zur Bundestagswahl 1987 gelang es ihr zwar, in nahezu allen Wahlkreisen Kandidaten aufzustellen, aber die Verbindung wurde nicht als Liste geführt, sondern nur mit einem einheitlichen Kennwort. Hinzu kam, dass explizit zur Wahl der Grünen oder der SPD mit der Zweitstimme aufgerufen wurde.

Zwischen 1990 und 2013 schwankte die Anzahl parteiloser Kandidaten zwischen 37 und 166. Tendenziell steigt sie an, ohne allerdings das Plateau von 1987 noch einmal erreicht zu haben (siehe Abbildung 1). Von einer neuen Phase zu sprechen, scheint angesichts der geringen Bedeutung übertrieben; eine gewisse Fluidität in Anzahl und Organisationsgrad ist aber zu beobachten.

Das Verhältnis von organisierten zu „echten“ Einzelbewerbern lässt sich nicht pauschalieren. Ins Auge fällt, dass jedes Mal, wenn die Anzahl der unabhängigen Kandidaten besonders hoch war, die Zahl der Organisierten den größten Anteil daran hatte. 2009 stellten etwa das „Willi-Weise-Projekt“ (62) und die Gruppe „Für Volksentscheide“ (21) zusammen

33 Vgl. Rudolf van Hüllen, Die Friedensliste (Frieden), in: Frank Decker / Viola Neu (Hrsg.), Handbuch der deutschen Parteien, Wiesbaden 2013, S. 298 - 299. 


\begin{tabular}{|c|c|c|c|}
\hline \multicolumn{3}{|c|}{ Tabelle 1: Anzabl der Einzelbewerber bei den Bundestagswablen seit 1949 } \\
\hline Bundestagswahl & $\begin{array}{c}\text { unorganisierte (,echte } \\
\text { Einzelbewerber }\end{array}$ & $\begin{array}{c}\text { organisierte } \\
\text { Einzelbewerber }\end{array}$ & Gesamt \\
\hline 1949 & 36 & 81 & 117 \\
1953 & 2 & 4 & 6 \\
1957 & 1 & 1 & 2 \\
1961 & 2 & 4 & 6 \\
1965 & 2 & 0 & 2 \\
1969 & 6 & 13 & 19 \\
1972 & 5 & 2 & 7 \\
1976 & 13 & 4 & 17 \\
1980 & 9 & 0 & 9 \\
1983 & 8 & 1 & 9 \\
1987 & 15 & 246 & 261 \\
1990 & 27 & 24 & 51 \\
1994 & 23 & 14 & 37 \\
1998 & 75 & 30 & 105 \\
2002 & 51 & 9 & 60 \\
2005 & 55 & 5 & 60 \\
2009 & 59 & 107 & 166 \\
2013 & 62 & 19 & 81 \\
\hline Summe & 451 & \multicolumn{2}{c}{} \\
\hline Quelle: Zusammenstellung durch Valentin Schröder nach den Angaben des Statistischen Bundesamtes \\
beziehungsweise des Bundeswahlleiters, a.a.O. (Fn. 37).
\end{tabular}

die Hälfte der insgesamt 166 Bewerber. Bei Wahlen, in denen es keine vergleichbaren Projekte gibt, überwiegt die Zahl der unorganisierten Kandidaten (siehe Tabelle 1).

Mit der Wiedervereinigung lassen die Unterschiede in der Politischen Kultur zwischen Ost und West, besonders ein unterstellter stärkerer Anti-Parteien-Affekt in den neuen Ländern, es wahrscheinlich erscheinen, dass es in Ostdeutschland mehr Einzelbewerber gibt. ${ }^{34}$ Der Blick auf die absoluten Zahlen in Tabelle 2 bestätigt diese Vermutung zunächst nicht. Wenn man jedoch bedenkt, dass die neuen Länder zwischen einem Fünftel und einem Sechstel der Wahlkreise des Bundesgebietes ausmachen, ist ihre Zahl im Vergleich relativ groß. 35

Der Anteil der Parteilosen an den Erstimmen in ihren jeweiligen Wahlkreisen ist zumeist klein; nur bei der ersten Bundestagswahl gelang es drei von ihnen, Direktmandate zu erringen. Kandidaten mit über zehn Prozent sind eine Seltenheit (siehe Tabelle 3). Exemplarisch kann an dieser Stelle auf einen der Dauerbewerber geschaut werden. In ihm verbindet sich

34 Die Forschung hierzu ist umfangreich, als Beleg daher nur eine größere empirische Studie von Gert Pickel / Detlef Pollack / Olaf Müller / Jörg Jacobs (Hrsg.), Osteuropas Bevölkerung auf dem Weg in die Demokratie. Repräsentative Untersuchungen in Ostdeutschland und zehn osteuropäischen Transformationsstaaten, Wiesbaden 2006.

35 Für 2013 entfielen von den 299 Wahlkreisen 56 auf die neuen Länder und den Osten Berlins. Damit traten hier 0,54 Einzelbewerber pro Wahlkreis an, in den alten Ländern lediglich 0,23. 


\begin{tabular}{|c|c|c|}
\hline Bundestagswahl & Einzelbewerber-Ost Gesamt & Einzelbewerber-West Gesamt \\
\hline 1990 & 9 & 42 \\
\hline 1994 & 7 & 30 \\
\hline 1998 & 25 & 80 \\
\hline 2002 & 13 & 47 \\
\hline 2005 & 22 & 41 \\
\hline 2009 & 53 & 119 \\
\hline 2013 & 30 & 55 \\
\hline Summe & 159 & 414 \\
\hline
\end{tabular}

das schwer in Parteien zu integrierende „Querkopfgehabe“, eine eigene politische Agenda und ideeller Partizipationswille.

Helmut Palmer - der „Remstal-Rebell“ - war bereits 1972 im Wahlkreis 177 (Waiblingen) angetreten und hatte 7.104 Erststimmen (4,9 Prozent) erhalten. Daneben bemühte er sich um Landes- und Kommunalparlamentssitze sowie 250-mal, ein Bürgermeisteramt zu erlangen. ${ }^{36}$ Nach seiner „erfolgreichen“ Zeit in den 1980er Jahren (siehe Tabelle 3) gelang es ihm in Waiblingen 1994 noch einmal, 13.020 Stimmen und damit 7,8 Prozent zu holen, und 1998 erhielt er 7.898 Stimmen (4,7 Prozent) im Wahlkreis 172 (Schwäbisch Hall - Hohenlohe). ${ }^{37}$ Damit ist Palmer einer von zwei unabhängigen Kandidaten, der nicht nur

\begin{tabular}{|c|c|c|c|c|}
\hline \multicolumn{6}{|c|}{ Tabelle 3: Einzelbewerber mit über zehn Prozent Erststimmen seit 1953 } \\
\hline BTW & Name & Wahlkreis (Nummer) & Stimmen & $\begin{array}{c}\text { Anteil } \\
\text { (in Prozent) }\end{array}$ \\
\hline 1953 & Richard Freudenberg & Mannheim-Land (180) & 15.709 & 20,8 \\
1969 & Wilhelm Daniels & Bonn (63) & 29.895 & 20,1 \\
1983 & Helmut Palmer & Göppingen (167) & 28.456 & 19,8 \\
1987 & Helmut Palmer & Waiblingen (168) & 31.625 & 19,2 \\
1987 & Franz Handlos & Deggendorf (213) & 17.523 & 17,2 \\
1990 & Helmut Palmer & Reutlingen (193) & 16.148 & 11,3 \\
2005 & Martin Hohmann & Fulda (176) & 39.545 & 21,5 \\
2005 & Konrad Dippel & Weiden (236) & 17.944 & 13,6 \\
2009 & Konrad Dippel & Weiden (236) & 17.196 & 14,1 \\
\hline Quelle: Eigene Darstellung nach den Angaben des Statistischen Bundesamtes, des Bundeswahlleiters und \\
Valentin Schröder, a.a.O. (Fn. 37).
\end{tabular}

36 Siehe hierzu Hermann-Arndt Riethmüller, Nachruf auf Helmut Palmer, http://www.helmut-palmer.de/ (Abruf am 4. Februar 2014); Tübingen Universitätsstadt (Hrsg.), Oberbürgermeister: Lebenslauf, http://www.tuebingen.de/75.html\#2089 (Abruf am 4. Februar 2014).

37 Vgl. Valentin Schröder, Deutschland seit 1945. Bundestagswahlen. Ergebnisse der Einzelbewerber und Wählergruppen in den Wahlkreisen, Stand: 18. Dezember 2013, http://www.wahlen-indeutschland.de/bEZB.htm (Abruf am 27. Januar 2014). 
kontinuierlich angetreten ist, sondern für immerhin drei aufeinanderfolgende Wahlen in den Genuss der staatlichen Wahlkampfkostenerstattung gekommen ist. Sein Sohn, Boris Palmer, war von 2001 bis 2007 Mitglied des Landtags von Baden-Württemberg für Bündnis 90/Die Grünen und ist seit Januar 2007 Oberbürgermeister von Tübingen. ${ }^{38}$

\subsection{Alters-, Geschlechts- und Typenverteilung bei der Bundestagswahl 2013}

Um eine differenzierte Beschreibung der Bewerber zu ermöglichen, bietet sich eine qualitative Analyse der Wahlziele und von Presseberichten an. ${ }^{39}$ Exemplarisch wurden daher für die Bewerber zur Bundestagswahl 2013 Kennwort, Alter, Geschlecht, Beruf und, aus der Pressedokumentation abgeleitet, der Typ erhoben. ${ }^{40}$

Von 81 Bewerbern sind nur drei weiblich. Bei der Altersverteilung sind 81,5 Prozent über 40 Jahre, 58 Prozent über 50 Jahre und rund 28,5 Prozent über 60 Jahre alt. Jünger als 30 sind lediglich drei Kandidaten. Bei Bildung respektive Beruf fallen in aufsteigender Reihenfolge die Nennungen Student, Rentner, Selbständiger und Diplom-Ingenieur auf. ${ }^{41}$

Bei der Zuordnung der Bewerber zu den sechs oben entwickelten Typen zeigt sich folgendes Bild ${ }^{42}$ : Dominant ist der Typ Protest (27/79). Die Stellung gegen Gesetze, aktuelle politische Inhalte und wahrgenommene gesellschaftliche Missstände ist dabei die Triebkraft. An zweiter Stelle steht mit Alternativ/Reform (22/79) ein Typ, der ebenfalls einen Missstand benennt, aber anders als der erste auch mehr oder weniger konkrete Vorschläge zu dessen Behebung präsentiert. Eine Sonderform ist in dieser Hinsicht der Typ Direkte Demokratie (18/79). Dieser strebt nach mehr Bürgerbeteiligung und sieht hier die größte Chance zur Reform des politischen Systems. Anti-Parteien (4/79), Eigenwerbung (4/79) und Single-Issue (3/79) sind nicht häufig, aber in den wenigen Fällen, wo sie kodiert werden konnten, eindeutig. ${ }^{43}$ Eigenwerbung im Sinne von kommunalpolitischer

38 Vgl. Georg Löwisch, Das Rebellenkind, in: taz online vom 17. Oktober 2006, http://www.taz. de/1/archiv/archiv/?dig=2006/10/17/a0143 (Abruf am 4. Februar 2014). Im Oktober 2014 gelang ihm mit 62 Prozent der Stimmen die Wiederwahl. Vgl. Florian Gathmann, Grünen-Politiker Palmer. Überflieger mit Demutsdefizit, in: Spiegel online vom 20. Oktober 2014 http://www. spiegel.de/politik/deutschland/gruenen-politiker-palmer-ueberflieger-mit-demuts-defizita-998106.html (Abruf am 29. Oktober 2014).

39 Basis hierfür waren die Informationszusammenstellungen des Bundeswahlleiters; Valentin Schröder, a.a.O. (Fn. 37); Severin Tatarczyck, Liste unabhängiger Kandidaten bei der Bundestagswahl 2013, http://www.severint.net/2013/08/07/liste-unabhaengiger-kandidaten-bei-der-bundestagswahl-2013/ (Abruf am 29. Oktober 2014) und die Online-Recherche in Regional- und Lokalzeitungen.

40 Dabei ist der Wert der Presseerzeugnisse etwas zweifelhaft. In allen Fällen haben die kurzen Berichte oder Interviews nur als Bestätigung den aus Kennwort, Homepage, Facebook- und Twitter-Profilen gewonnenen Eindruck bestätigt.

41 Da es sich bei den Berufen wie allgemein üblich um Eigennennungen handelt und darüber hinaus in den meisten Fällen keine Daten zu den Personen vorliegen, ist eine genaue Auswertung nicht möglich.

$42 \mathrm{~N}=79$, weil zu zwei Bewerbern keine inhaltlichen Aussagen gemacht werden konnten. Das Kennwort war in beiden Fällen nichtssagend: Wählergruppe Falk und Freie Liste Kreis Düren.

43 Sehr eindrücklich ist hier eines der wenigen tiefergehenden Interviews mit einem Bewerber. Vgl. Kristina Koebe, „Wählt Wiechmann“. Interview mit Steffen Wiechmann, unabhängiger Rostocker Kandidat bei der Bundestagswahl 2013, in: Stadtgespräche, 20. Jg. (2014), H. 73, S. 8 - 11. 


\begin{tabular}{|c|c|c|c|c|}
\hline \multicolumn{5}{|l|}{ Tabelle 4: } \\
\hline Jahrgänge & Altersgruppe & Männer & Frauen & Summe \\
\hline 1935 bis 1943 & über 70 Jahre & 6 & 0 & 6 \\
\hline 1944 bis 1953 & über 60 Jahre & 16 & 1 & 17 \\
\hline 1954 bis 1963 & über 50 Jahre & 24 & 1 & 24 \\
\hline 1964 bis 1973 & über 40 Jahre & 18 & 1 & 19 \\
\hline 1974 bis 1983 & über 30 Jahre & 11 & 0 & 11 \\
\hline 1984 bis 1993 & über 20 Jahre & 2 & 0 & 2 \\
\hline 1994 & 19 Jahre & 1 & 0 & 1 \\
\hline Summe & & 78 & 3 & 81 \\
\hline
\end{tabular}

\begin{tabular}{|l|l|c|c|}
\hline \multicolumn{1}{|c|}{ Tabelle 5: Einzelbewerbertypen bei der Bundestagswahl 2013 } & \multicolumn{1}{|c|}{ Beschreibung } & $\begin{array}{c}\text { Häufigkeit } \\
\text { (N =79) }\end{array}$ & $\begin{array}{c}\text { Anteil } \\
\text { (in Prozent) }\end{array}$ \\
\hline Alternativ/Reform & $\begin{array}{l}\text { Andere Welt- und Ordnungsbilder sowie } \\
\text { Anpassungsideen für die Gesellschaft }\end{array}$ & 22 & 27,8 \\
Anti-Parteien & Gegen das Kartell der Parteien & 5 & 6,3 \\
Direkte Demokratie & Bürgerbeteiligung & 18 & 22,8 \\
Eigenwerbung & Bekanntmachung mit ökonomischen Motiven & 4 & 5,1 \\
Protest & $\begin{array}{l}\text { Grundsätzliche oder themenspezifische } \\
\text { Single-Issue }\end{array}$ & $\begin{array}{l}\text { Dagegensein } \\
\text { Einthemenausrichtung }\end{array}$ & 34,2 \\
\hline * Bei doppelter Typenvergabe wurde jeweils nur der erste in die Auszählung einbezogen. & 3,8 \\
\hline
\end{tabular}

Bekanntmachung der eigenen Person war als Typ aus den vorhandenen Daten nicht zu ermitteln.

Grundsätzlich kann gesagt werden, dass die abgeleiteten Leitmotive nicht exklusiv sind; vielmehr dienen Protest und die mehr oder weniger deutliche Stellung gegen Parteien und das durch sie ausgeübte Regierungshandeln als Hintergrundfolie.

Abschließend ist zu sagen, dass die wenigsten Einzelbewerber Mitglied in einer Partei waren. Die Ausnahmen - wie Siegfried Kauder - genießen dafür eine gewisse Bekanntheit und werden auch medial wahrgenommen. ${ }^{44}$ In einem Fall hat es sich gezeigt, dass der ehemalige Einzelbewerber im Jahr 2014 für die AfD aktiv wurde.

44 Vgl. Hugo Müller-Vog, Als Einzelkämpfer in den Bundestag, in: Cicero online vom 30. August 2013, http://www.cicero.de/berliner-republik/direktkandidaten-als-einzelkaempfer-den-bundestag/55594/seite/2 (Abruf am 29. Oktober 2014). 


\section{Erfolgsbedingungen und Hürden - Einzelbewerber als Protestventil?}

Was ist Erfolg für Einzelbewerber? ${ }^{45}$ Das oberste Ziel ist offensichtlich, ein parlamentarisches Mandat zu erringen. Dies ist seit der ersten Bundestagswahl nicht wieder gelungen ist. Damit ergibt sich ein Minimalziel: eine Wahlalternative zu den etablierten oder allgemein zu Parteien bereitzustellen. Dazwischen liegen, die Zulassung zur Wahl vorausgesetzt, persönliche, soziale, ökonomische und politische Motive. In Anbetracht der Tatsache, dass ein Wahlkampf neben den zeitlichen Ressourcen vor allem Geld kostet, muss das realistische Interesse von unabhängigen Kandidaten gemäß $\$ 49 \mathrm{~b}$ BWahlG sein, mindestens zehn Prozent der Erststimmen in ihrem Wahlkreis zu erhalten. Nur unter dieser Voraussetzung wird ihnen jede Wählerstimme mit 2,80 Euro vergütet. In dieser Hinsicht sind auch organisierte parteilose Kandidaten, die beispielsweise bundesweit mit demselben Kennwort antreten, letztlich Einzelkämpfer.

Vor diesem Hintergrund kann gesagt werden, dass der Aufstieg unter dem gleichbleibenden Banner des eigenen Namens und die kontinuierliche Arbeit als parteiloser Kandidat Wirkung zeigen können. So hat Andreas Beier im Wahlkreis Ulm bei den Bundestagswahlen zwischen 1998 und 2005 stets sein Stimmenergebnis der vorherigen Wahl übertroffen (siehe Tabelle 6). 2009 trat er dann erstmals unter einem Motto („Für Volksentscheide“), also als organisierter Einzelbewerber, an. Mit 2.329 Stimmen gelang ihm sein bestes Ergebnis. Im Jahr 2013 erweiterte er sein Kennwort auf „Für bürgernahe Demokratie und Volksentscheide / Danke für ihre Erststimme“. Zum einen war er damit wieder ein „echter“ parteiloser Kandidat, allerdings ging zum anderen sein Ergebnis um nahezu 1.000 Stimmen zurück.

Beiers Bemühungen als parteiloser Kandidat zeichnen sich durch Idealismus, aber begrenzten Zuspruch aus. Inwieweit ist er, ohne dass er dies programmatisch intendiert ${ }^{46}$, eine Projektionsfläche für Protestwahl? Der Stimmenverlust beim Wahlgang 2013 lässt sich möglicherweise auf das etwas sperrige Kennwort zurückführen. Geht man aber davon aus, dass Beier seinen Stimmenzuwachs bei den vorherigen Wahlen seiner steigenden Bekanntheit verdankt, scheint dies nur ein Teil der Erklärung zu sein. Ein Blick auf das Feld der Kandidaten für das Direktmandat zeigt zwei Unterschiede zu 2009: Die FW mit Horst Dürr und die AfD mit Werner Otto Greipel hatten beide einen Bewerber aufgestellt. Diese holten zwar nur 1,0 beziehungsweise 3,6 Prozent der Erststimmen, stellten aber eine Konkurrenz zu Beier dar. ${ }^{47}$ Beide Parteien sind ihrem Selbstverständnis nach Alternativen zu den etablierten Parteien, und besonders die AfD schöpfte 2013 Protestwähler ab. ${ }^{48} \mathrm{Im}$

45 Die folgenden Ausführungen nehmen die von Oskar Niedermayer zu Parteien angestellten Überlegungen auf und wenden sie in einem ersten Aufriss auf Einzelbewerber an. Siehe hierzu ders., Erfolgsbedingungen neuer Parteien im Parteiensystem am Beispiel der Piratenpartei Deutschland, in: ZParl, 41. Jg. (2010), H. 4, S. 838 - 854; ders., Einleitung: Erfolgsbedingungen neuer Parteien im Parteiensystem, in: ders. (Hrsg.), Die Piratenpartei, Wiesbaden 2013, S. 7 - 14.

46 Vgl. Martin Reiher, Einzelbewerber zur Bundestagswahl - ein aussichtsloser Kampf?, http://www. wahlschlepper.net/einzelbewerber-zur-bundestagswahl-aussichtsloser-kampf/ (Abruf am 31. Januar 2014).

47 Vgl. Stadt Ulm (Hrsg.), Bundestagswahl 2013: Erststimmenergebnis (Endergebnis). Wahlkreis 291 Ulm, http://www.wahl.ulm.de/bt2013/Erst/bt2013erst.html (Abruf am 2. Februar 2014).

48 Eine erste Analyse zur AfD findet sich bei Richard Gebhardt, Eine „Partei neuen Typs“? Die „Alternative für Deutschland“ (AfD) vor den Bundestagswahlen, in: Forschungsjournal Soziale Bewegungen, 26. Jg. (2013), H. 3, S. 86 - 91; Rüdiger Schmitt-Beck, Euro-Kritik, Wirtschaftspessimismus und Einwanderungsskepsis: Hintergründe des Beinah-Wahlerfolges der Alternative für 


\begin{tabular}{|c|c|c|c|c|c|}
\hline \multicolumn{6}{|c|}{ Tabelle 6: Auf und ab des Einzelbewerbers Andreas Beier (Wahlkreis Ulm) } \\
\hline & 1998 & 2002 & 2005 & 2009 & 2013 \\
\hline $\begin{array}{c}\text { Erststimmen } \\
\text { (absolut) }\end{array}$ & 820 & 1.459 & 2.067 & 2.329 & 1.344 \\
\hline $\begin{array}{c}\text { Erststimmen } \\
\text { (in Prozent) }\end{array}$ & 0,5 & 0,9 & 1,2 & 1,5 & 0,8 \\
\hline Kennwort & $\begin{array}{c}\text { Andreas } \\
\text { Beier }\end{array}$ & $\begin{array}{c}\text { ANDREAS } \\
\text { BEIER }\end{array}$ & Andreas Beier & $\begin{array}{c}\text { Für Volks- } \\
\text { entscheide }\end{array}$ & $\begin{array}{c}\text { Für bürgernahe Demokratie } \\
\text { und Volksentscheide / } \\
\text { Danke für ihre Erststimme }\end{array}$ \\
\hline Quelle: Eigene Darstellung nach den Daten des Bundeswahlleiters. \\
\hline
\end{tabular}

Umkehrschluss und aus der obigen Analyse entsteht der Eindruck, dass Einzelbewerber durchaus als Protestventil wirken können. Allerdings werden Parteien auch in dieser Funktion vom Wahlvolk als geeigneter angesehen als Einzelpersonen.

\section{Fazit: "Hoffnung ist wie Zucker im Tee: zwar klein, aber sie versüßt“}

Die Dominanz der Parteien in der Organisation des Wählerwillens in Deutschland wird an der beiläufigen Behandlung von Einzelbewerbern deutlich. Zusätzlich ergeben sich aus der hohen Erfolgshürde Schwierigkeiten bei der Refinanzierung der aktiven Partizipation außerhalb von Parteien. Zu diesem Faktor kommt, dass unabhängige Kandidaten taktische Nachteile im Recht und ihrer Organisationen haben, was einen Einzug in den Bundestag bisher unterbunden. Gleichzeitig ist es ein fester Bestandteil der wissenschaftlichen Forschung und der populären Medien, neue Partizipationsformen im Gefolge der Digitalen Revolution und der oben angedeuteten Distanz zwischen Bürgern und Parteien zu thematisieren. ${ }^{49}$ Mit den global vernetzten Protestphänomenen der letzten Jahre sind einige Enthusiasten gar auf den Gedanken gekommen, dass im politischen Raum zukünftig nicht mehr Parteien, sondern eine möglicherweise internationalisierte Zivilgesellschaft dominieren werde. ${ }^{50}$

Deutschland (AfD) bei der Bundestagswahl 2013, in: ZParl, 45. Jg. (2014), H. 1, S. 94 - 112; zum Protestcharakter der AfD in der Auswertung der Bundestagswahl siehe Daniel Deckers, AfD scheitert an der Ablehnung durch Frauen, FAZ vom 29. Januar 2014, S. 2. Weiterführende Analysen im Nachgang der Erfolge bei der Europa- und den ostdeutschen Landtagswahlen 2014 finden sich in Christian Nestler / Jan Rohgalf, Eine deutsche Angst - Erfolgreiche Parteien rechts von der Union. Zur AfD und den gegenwärtigen Gelegenheitsstrukturen des Parteienwettbewerbs, in: ZfP, 61. Jg. (2014), H. 4 (im Erscheinen).

49 Vereinfacht unter dem Slogan „Die neue Macht der Bürger“ ist vor allem der Gedanke des sozialen Unternehmertums für die zukünftige Entwicklung der - weiterhin parlamentarischen - Demokratie in Deutschland einbezogen. Siehe Stine Marg / Lars Geiges / Felix Butzlaff / Franz Walter (Hrsg.), Die neue Macht der Bürger. Was motiviert die Protestbewegungen?, Reinbek bei Hamburg 2013; Saskia Richter, Parteigründungen, Wutbürger und soziales Unternehmertum. Krisen als Treiber neuer Formen politischer Partizipation, in: Oskar Niedermayer (Hrsg.), a.a.O. (Fn. 16), S. 157 - 175; dies., Politische Partizipation und soziale Bewegung in sich wandelnden Gesellschaften. Von Bürgerinitiativen zu Online-Netzwerken, in: Marianne Kneuer (Hrsg.), Das Internet: Bereicherung oder Stressfaktor für die Demokratie?, Baden-Baden 2013, S. 145 - 171.

50 Vgl. Geseko von Lüpke, Parteilos glücklich. Essay über das Entstehen einer neuen Form der Demokratie, in: Natur + Kosmos, 6. Jg. (2004), H. 6, S. $50-51$. 
Resümierend kann gesagt werden, dass es für die eingangs aufgeworfene Frage nach der Entwicklung der Einzelbewerber von Interesse ist, dass der Anteil jener mit mehr als einem Prozent der Erststimmen in ihrem jeweiligen Wahlkreis von 35 zwischen 1953 bis einschließlich 1994 auf 58 von 1998 bis 2013 angestiegen ist. ${ }^{51}$ In bescheidenem Umfang kann damit die These auch für Deutschland als bestätigt gelten, dass die Bedeutung von Einzelbewerbern gestiegen ist.

Deutlich ist weiterhin geworden, dass unabhängige Kandidaten eine Reihe von alternativen Zielsetzungen mit dem Bundestagswahlkampf verbinden. Solche Aktivitäten können, besonders wenn sie auf vernachlässigte Problemlagen aufmerksam machten, eine Bereicherung für die Gesellschaft und den Parteienwettbewerb sein. Die Funktion als Protestventil erfüllen sie eher ungewollt, da ohne Ernsthaftigkeit im Regelfall kein kosten- und zeitintensiver Mandatserwerb betrieben wird. Hinzu kommt, dass Parteien als Protestvehikel beim Wähler ein ungleich größeres Interesse hervorrufen.

Die Arena, in der Einzelbewerber in Deutschland die größte Rolle spielen - die Kommunalwahlen -, wurde hier nur gestreift. Der Vergleich mit der Bundesebene bietet sich in vielerlei Hinsicht nicht an. So ist es Konsens, dass die Vorrangstellung der Parteien in den Kommunen hinter die Gestaltungsbemühungen der Mandatsträger zurücktritt. ${ }^{52}$ Der Vergleich zu Landtagswahlen scheint dagegen von einigem Interesse. Hier dürften die Erfolgsaussichten prima facie größer sein. Die Wahlergebnisse der letzten Jahre widersprechen aber dieser Annahme. „Echte“ Einzelbewerber sind genauso wie auf der Bundesebene zahlreich, aber chancenlos. ${ }^{53}$ Im Detail kann hier erst ein Blick auf die Landtagswahlen in allen Bundesländern seit 1949 ein differenzierteres Bild ergeben. Auch dabei ist und bleibt die Hürde eine adäquate Datenerhebung über das Wahlergebnis hinaus, denn Internetseiten o.ä. sind zumeist kurze Zeit nach der Wahl schon offline und Zeitungsarchive im Regelfall nicht kostenlos zugänglich.

Auch ein internationaler Vergleich könnte, nicht zuletzt vor dem Hintergrund der deutlich größeren Erfolge parteiloser Bewerber beispielsweise in Irland, von Interesse sein. ${ }^{54}$ Unterschiedliche Politische Kulturen messen der Parteigebundenheit ihrer Parlamentarier und zum Teil ihrer Präsidenten einen unterschiedlichen Stellenwert bei. Ein Abweichen von der Dominanz der Parteien im politischen System ist aber nicht absehbar und wäre mit Blick auf die Repräsentations- und Interessenartikulationsfunktion auch unlogisch. Doch der Parteienstaat in Deutschland hat anders als das Grundgesetz keine Ewigkeitsklausel; neue Partizipations- und Repräsentationsformen sind als Teil der Systementwicklung nicht ausgeschlossen.

51 Anknüpfend an den Enthusiasmus von Geseko von Lüpke wäre ein unmittelbarer Anreizvorschlag für eine bessere Motivation bei den Einzelbewerbern und damit möglicherweise auch für die Partizipation allgemein eine Absenkung der Wahlkampfkostenerstattungsgrenze von zehn auf eine von hundert Stimmen im Wahlkreis. In Tabelle 3 waren die sechs Bewerber, die bei neun Gelegenheiten den Sprung über die Hürde geschafft haben, abgebildet. Sie hatten nach dem 2013 gültigen Erstattungssatz von 2,80 Euro zusammen seit 1953 einen Anspruch auf 599.315,80 Euro. Bei einer entsprechend niedrigeren Hürde beliefe sich die zusätzliche Summe auf 543.827,20 Euro. In den Genuss wären statt sechs 84 Bewerber gekommen.

52 Siehe als Überblick Everhard Holtmann, Parteien auf der kommunalen Ebene, in: Oskar Niedermayer (Hrsg.), a.a.O. (Fn. 4), S. $791-815$.

53 Vgl. Christian Nestler, a.a.O. (Fn. 30), S. 180.

54 Vgl. Nicole Bolleyer / Liam Weeks, a.a.O. (Fn. 27), S. 14 - 15. 